Pacific Journal of Mathematics

FINITE PRIMES OF FIELDS AND COMPLETIONS 


\section{INFINITE PRIMES OF FIELDS AND COMPLETIONS}

\section{K. Harrison AND HoYt D. WARNer}

The notion of infinite prime in a ring with identity, defined in the first author's memoir "Finite and infinite primes for rings and fields" (A.M.S Memoir \#68), is studied in fields. Extending results of $R$. Baer and D. W. Dubois, each infinite prime $P$ of a field $F$ is shown to determine a complex place $\phi_{P}$ of $F$ such that $\phi_{P}(P)$ is the set of nonnegative reals in $\phi_{P}(F)$, and is an infinite prime of $\phi_{P}(F)$. The collection of all infinite primes $P$ of $F$ determining the same $\phi$ and $\phi(P)$, is shown to be describable, in an almost purely multiplicative way, in terms of certain groups determined by $\phi$ and $\phi(P)$. Using these theorems a notion of completion of a field at a finite or infinite prime is given, generalizing the classical notion for the prime divisors of a number field. These completions are characterized as certain linearly compact fields and are shown to be in general unique only when $P$ is real (i.e., $\phi_{P}(F)$ is contained in the reals). For a fixed prime $P$ of $F$, the set of elements of $F$ which are squares in every completion of $F$ at $P$ is calculated.

$A$ characterization of number rings is given and examples of pathology in infinite primes are indicated.

An arithmetic of rings has been introduced in [11]. A notion of a prime is given which essentially reduces, in both number fields and their rings of integers, to the usual primes-both the finite and infinite. In this paper we examine closely the notion of an infinite prime. (The words "infinite" and "finite" are used formally as in number theory and have nothing to do with "number of elements".) We restrict attention mainly to commutative rings (for noncommutative rings see [16], [21] $)^{1}$; Proposition 0 below essentially reduces us to the study of fields.

We make the following definitions and conventions. All rings have an identity 1 and are assumed commutative unless otherwise stated. If $M$ is an additive subgroup of a ring $R$, we let

$$
A_{M}=\{a \in R \mid a M \subseteq M, M a \sqsubseteq M\} \text {. }
$$

For subsets $A, B$ of a ring $R$ we define $A+B=\{a+b \mid a \in A, b \in B\}$, likewise for $A \cdot B, A-B ;-A=\{-a \mid a \in A\}$. Let $A \backslash B=\{a \in A \mid a \notin$ $B\}, A^{-}=A \cap-A, A^{+}=A \backslash A^{-}, A^{-1}=\left\{a^{-1} \mid a \in A, a \in\right.$ units of $\left.R\right\}$. For a field $F, F^{\times}$denotes the multiplicative group of nonzero elements of $F$. $\boldsymbol{N}, \boldsymbol{Z}, \boldsymbol{Q}, \boldsymbol{R}, \boldsymbol{C}, \boldsymbol{P}$ denote the positive integers, integers, rationals,

${ }^{1}$ See also W. H. Reynolds, $A$ note on embedding a partially ordered ring in a division algebra, P. A. M. S. 37 (1973), 37-41. 
reals, complexes, nonnegative reals, respectively.

By a preprime $S$ of a (not necessarily commutative) ring $R$ we mean a subset $S \neq \varnothing$ of $R$ such that $S+S \subseteq S, S \cdot S \subseteq S,-1 \notin S$. A prime $P$ of $R$ is a maximal preprime; $P$ is called finite if $1 \notin P$ (equivalent to $-P \subseteq P$ ), otherwise infinite. A preprime $S$ containing 0 is called conic if $S^{-}=\{0\}$; the standard example is an order $S$ of $R$, by which we mean $S$ is the nonnegative cone of a linear ordering of the ring $R$. For example, $\boldsymbol{P}$ is an order (and infinite prime) of $\boldsymbol{R}$, and also an infinite prime of $\boldsymbol{C}$. For any unproven assertion about primes, see [11].

We generalize the ring of bounded elements and ideal of infinitesimals for an order and define, for any subset $S$ of $R$ closed under addition and multiplication,

$$
\begin{aligned}
& B_{S}=\{a \in R \mid m \cdot 1+a \in S, m \cdot 1-a \in S, \text { for some } m \in N\} \\
& J_{S}=\{a \in R \mid m \cdot 1+Z \cdot a \leqq S, \text { for some } m \in N\}
\end{aligned}
$$

and, if $J_{S}$ is an additive subgroup of $R$, we define $A(S)=A_{M}$ for $M=$ $J_{S} \cdot \quad B_{P}$ is a subring of $R$ and $J_{P}$ is an ideal of $B_{P}$ whenever $P$ is a prime of $R\left(J_{P}=P, B_{P}=Z \cdot 1+P\right.$ if $P$ is finite; $P$ infinite implies $2 \cdot P=P$ by $[11,1.6]$ so $J_{P}=\{a \in R \mid 1+Z a \subseteq P\}$ and the assertion follows by calculation, see [7]). We remark that it is straightforward to show that an order $S$ of a ring $R$ is a prime of $R$ if and only if $J_{S}$ contains no nonzero two sided ideals of $R$.

We call a prime $P$ of a ring $R$ archimedean if $P \subseteq B_{P}$; thus all finite primes are archimedean (note: by [11, 1.6] our definition of archimedean coincides with that of [11]). A non-archimedean order in a field (i.e., the cone of a non-archimedean ordering) is an example of a non-archimedean prime. It follows from [11, Prop. 1.3 and Theorem 1.7] that any archimedean prime (finite or infinite) of a field determines a place into a "nice" field: a locally finite field (i.e., an absolutely algebraic field of nonzero characteristic) if $P$ is finite, the complex numbers if $P$ is infinite. Baer [1] and Dubois [10] have results extending this to, respectively, non-archimedean orders and to non-archimedean infinite primes close to orders, called "modes" ( $P$ is a mode if $P / P^{-}$is an order of $\left.A_{P^{-}} / P^{-}\right)$. Theorem 1.1 below extends this result to arbitrary non-archimedean infinite primes of fields, i.e., to arbitrary infinite primes of fields, and shows that there is always an archimedean infinite prime associated with any nonarchimedean infinite prime $P$, namely (cf. [10])

$$
\operatorname{Arch} P=\left\{a \in B_{P} \mid n \cdot 1+N \cdot a \subseteq P \text { for some } n \in N\right\} \text {. }
$$

This, plus the result of $\S 2$ that $T=\operatorname{Arch} P$ determines all the $P$ 's such that $\operatorname{Arch} P=T$, indicates that in studying primes one can restrict attention to archimedean primes, at least in studying fields. 
For our purposes we may turn attention to fields because of the following result.

Proposition 0. Let $P$ be a prime of a commutative ring $R$. Let $Q=\{x \in R \mid x \cdot R \cong P\}$. Then $Q$ is a prime ideal of $R$. Let $F$ be the quotient field of $R / Q$. Then there exists a unique prime $S$ of $F$ with $P \cdot 1_{F} \subseteq S . \quad S$ is infinite if and only if $P$ is infinite.

Explicitly, if $U(P)$ denotes the set of $u \in R$ with $P=\{x \in R \mid x \cdot u \in$ $P\}$, then $S$ is the set of all $(a+Q) \cdot(u+Q)^{-1}$ with $a \in P, u \in U(P)$. $U(P)$ is $P^{+}$if $P$ is infinite and is $\left\{a \in R \mid a^{n}-1 \in P\right.$ for some $\left.n \in N\right\}=$ $A(P) \backslash P$ if $P$ is finite.

Proof. That $Q$ is a prime ideal follows from [11, 2.2]. Next observe that the last sentence (structure of $U(P)$ ) follows from [11, 1.6] and [11, 2.7]. Let $S$ be the set of all $(a+Q) \cdot(u+Q)^{-1}$ in $F$ with $a \in P, u \in U(P)$. Using [11, 2.2] one checks that every element $t$ in $F$ can be written as $(x+Q) \cdot(u+Q)^{-1}$ with $x \in R, u \in U(P)$, and that this $t$ is in $S$ if and only if $x \in P$. With this, one checks $S$ is a preprime; thus it is contained in a prime $T$ of $F$. This set $\{x \in R \mid x+$ $Q \in T\}$ is a preprime of $R$ containing $P$, so it equals $P$. Using the structures of $U(T)$ and $U(P)$ and considering the cases $P$ finite and $P$ infinite separately, one checks $\{x \in R \mid x+Q \in U(T)\}=U(P)$. Writing $t \in T$ as above, one has $(u+Q) \cdot t=x+Q$ for some $u \in U(P), x \in R$. Then $u+Q \in U(T)$ so $(u+Q) \cdot t \in T$; thus $x+Q \in T$ and $x \in P$. Hence $t \in S$, and $T=S$. The remaining assertions are easily checked.

1. Structure of an infinite prime in a field. The main result of this section is the following theorem (see above for definitions).

THEOREM 1.1. Let $P$ be an infinite prime of the field $F$. Then $A(P)$ is a valuation ring of $\boldsymbol{F}$ with $J_{P}$ its maximal ideal, and $\boldsymbol{P}_{P}=$ $\left[(P \cap A(P)) \cup J_{P}\right] / J_{P}$ is a conic archimedean infinite prime of the residue field $k_{P}=A(P) / J_{P}$.

There is a complex place $\phi_{P}$ of $F$ with domain $A(P)$, kernel $J_{P}$, such that $\phi_{P}(P)=\phi_{P}(F) \cap P$, so $\phi_{P}$ induces an isomorphism of $k_{P}$ into $\boldsymbol{C}$ sending $\boldsymbol{P}_{P}$ onto $\phi_{P}(F) \cap \boldsymbol{P}$. The restriction $\left.\phi_{P}\right|_{B_{P}}$ is unique (however, $\phi_{P}$ need not be unique).

Arch $P=\phi_{P}^{-1}(\boldsymbol{P})=(P \cap A(P)) \cup J_{P}$ and is an archimedean infinite prime of $F$ (with $\left.J_{\mathrm{Arch} P}=J_{P}, k_{\mathrm{Arch} P}=k_{P}, \boldsymbol{P}_{\mathrm{Arch} P}=\boldsymbol{P}_{P}\right)$.

REMark. If $P$ is a mode then $B_{P}=A(P)$, so Dubois' result [10, Theorem II] is a corollary. The proof that $J_{P}$ is the kernel of a place depends on techniques inspired by Dubois, see Lemma 1.2 below. That the place is complex depends crucially on an application of [11, Prop. 1.7] to a ring which is not necessarily a field. We shall call an 
infinite prime of a field real if the place $\phi_{P}$ is into the reals, otherwise we call $P$ complex. This accords with algebraic number theory terminology, see $[11, \S 3]$. One can show that $P$ is real if and only if $A(P)=B_{P}$, or, if and only if $B_{P}$ is a valuation ring of $F$.

We assemble the technical results needed for this and the next section in the following lemma. For simplicity we write "subgroup" for "multiplicative subgroup". By a "valuation pair" of a commutative ring $R$ we mean, following Manis [15], a pair $(A, M)$ where $A$ is a subring of $R, M$ a prime ideal of $A$ and $x \in R \backslash A$ implies there is $y \in$ $M$ with $x y \in A \backslash M$. When $R$ is a field these are the valuation rings of $R$ together with their maximal ideals. See [15] or [11, p. 18] for facts about valuation pairs which we shall use. We write value groups additively.

TeChnical Lemma 1.2. Let $F$ be a field, $S$ a subset of $F$ with 0 and 1 in $S$. The following conditions are equivalent.

(1) $S$ is an infinite prime of $F$;

(2) $\left(A_{S^{-}}, S^{-}\right)$is a valuation pair of $F$ and $S^{+}$is a maximal additively closed subgroup of $A_{S^{-}} \mid S^{-}$.

(3) $\left(A_{S^{-}}, S^{-}\right)$is a valuation pair of $F$ and if $(A, M)$ is a valuation pair of $F$ with $M \supseteqq S^{-}$, the following are equivalent:

(a) $M \subseteq J_{s}$, or equivalently, $1+M \leqq S$,

(b) $S^{+} \cap(A \backslash M)$ is additively closed,

(c) $S^{+} \cap(A \backslash M)$ is a maximal additively closed subgroup of $A \backslash M$;

(4) $\left(A_{S^{-}}, S^{-}\right)$is a valuation pair of $F$ and there exists a valuation pair $(A, M)$ of $F$ with $M \supseteqq S^{-}$such that, letting $G=S^{+} \cap(A \backslash M)$,

(a) $G$ is a maximal additively closed subgroup of $A \backslash M$,

(b) $S^{+}$is maximal among all subgroups $H$ of $A_{S^{-}} \backslash S^{-}$satisfying $H \cap(A \backslash M)=G$.

Moreover, if (1) holds, then (a), (b) of (4) hold for every valuation pair $(A, M)$ of $F$ with $S^{-} \leqq M \leqq J_{S}$.

Proof. See the Appendix.

The following result, which inspired part of the above lemma, is an immediate consequence of it.

Corollary 1.3. (Dubois $[10,4.4])$ Let $P$ be a conic preprime of the field $F$ with $1 \in P$. Then $P$ is a prime if and only if $P^{+}$is a maximal additively closed subgroup of $F^{\times}$. Hence any conic preprime of $F$ containing 1 is contained in a conic prime of $F$ (if char $(F)=$ 0 , any conic preprime of $F$ is contained in a conic prime).

Proof of Theorem 1.1. Since $J_{P}$ is an ideal of $B_{P}, P_{0}=P \cap B_{P}$ a preprime of $B_{P}$ implies $P_{0}+J_{P}$ is also a preprime, so contained in 
a prime $T$ of $B_{P} . \quad B_{P} \subseteq P_{0}-P_{0} \subseteq T-T$, so $T^{-}$is a prime ideal of $B_{P}=T-T$ by [11, Prop. 2.5] (applied to $T$ and $B_{P}$ ). $T^{+} \subseteq B_{P} \backslash T^{-}$ is then closed under addition and multiplication. By [15, Prop. 1] there is a valuation pair $(A, M)$ of $F$ such that $A \supseteqq B_{P}$ and $M \cap B_{P}=$ $T^{-}$. We claim that $P^{+} \cap(A \backslash M)$ is additively closed, so that by (3) of Lemma 1.2, $M \cong J_{P}$ and hence $M=T^{-}=J_{P}, A=A(P)$, proving the first assertion of 1.1. To prove the claim it suffices to show $P^{+} \cap(A \backslash M)$ is contained in $T^{+} \cdot\left(T^{+}\right)^{-1}$, an additively closed subset of $A \backslash M$. If $a \in P^{+} \cap(A \backslash M), a=(1-\alpha) / \alpha$ for $\alpha=(1+\alpha)^{-1}$ and by an argument in the proof of [11, Prop. 3.5], and valuation theory, $\alpha$ and $1-\alpha$ are each in $P_{0}$ and not in $M$, so they are in $T^{+}$, proving the claim. Moreover, then by (3) (b) of Lemma 1.2, it follows that $P^{+} \cap\left(A(P) \backslash J_{P}\right)=T^{+}\left(T^{+}\right)^{-1} \supseteq T^{+}$.

We now apply [11, Prop. 1.7] to the prime $T$ of the ring $B_{P}(T$ is easily checked to be archimedean) to get a unique homomorphism $\psi: B_{P}=T-T \rightarrow \boldsymbol{R}$ with $\psi^{-1}(\boldsymbol{P})=T, \operatorname{ker} \psi=T^{-}$. With this we can show that in fact $P^{+} \cap\left(A(P) \backslash J_{P}\right) \subseteq T^{+}$. For, if $\alpha \in P^{+} \cap\left(A(P) \backslash J_{P}\right)$ and $\alpha=(1+\alpha)^{-1}$ as above, then $\alpha \in T^{+}$, so $\psi(\alpha)>0$ and there exists $n \in N$ with $n \psi(\alpha)-\psi(1-\alpha)>0$, i.e., $n \cdot \alpha-(1-\alpha) \in T^{+} \subseteq P$. Hence (as $\left.\alpha^{-1} \in P\right) \quad n \cdot 1-a=n \cdot 1-(1-\alpha) / \alpha \in P$ and $a \in P \cap B_{P}=P_{0}$, so $a \in$ $P_{0} \mid J_{P} \subseteq T^{+}$. Therefore, $P^{+} \cap\left(A(P) \backslash J_{P}\right)=T^{+}$and $T$ is an infinite (archimedean) prime of $F$ by Lemma 1.2. (Apply (3) (c) to $P$ and the pair $(A, M)=\left(A_{T^{-}}, T^{-}\right)$; then $T$ satisfies (2), hence (1) of the Lemma.) It follows that $\bar{T}=T / T^{-}=T / J_{P}$ is a conic, archimedean infinite prime of $k_{P}=A(P) / J_{P}$; since (using the $T^{+}$calculation) $\bar{T}=P_{P}$, this completes proof of the first sentence of 1.1. Next, $k_{P}$ is algebraic over the subring $\bar{T}-\bar{T}$ (as $-1 \in \bar{T}[c]$ for any $c \notin \bar{T}$ ), which is isomorphic to a subring of $\boldsymbol{R}$. Hence, $k_{P}$ is isomorphic to a subfield of $\boldsymbol{C}$ by an isomorphism $\bar{\phi}$ such that $\bar{\phi}^{-1}(P)=\bar{T}=P_{P}$. Now let $\phi_{P}$ be $\bar{\phi}$ composed with the place of $F$ onto $k_{P}$. Then $\phi_{P}(P \cap A(P))=\bar{\phi}\left(\boldsymbol{P}_{P}\right)=\phi(F) \cap \boldsymbol{P}$, as required. These properties and [11, Prop. 1.7] imply uniqueness of $\left.\phi_{P}\right|_{B P}$; note $B_{P}=\phi_{P}^{-1}(R)$. To conclude the proof, we show $T=$ Arch $P$. Arch $P \supseteqq T$ is easily checked (as $T^{-}=J_{P}$ and $T^{+}=P \cap$ $\left(A(P) \backslash J_{P}\right) \cong B_{P}$ are each contained in Arch $P$ from its definition). Conversely, $a \in \operatorname{Arch} P$ implies $a \in B_{P}=B_{T}$ and $1+N a \subseteq P$; hence $1+N a \subseteq P \cap B_{P} \subseteq T$ and $a \in$ Arch $T$. But Arch $T=T$ by [11, Lemma A5] (see [9] for a simplified proof), so Arch $P \subseteq T$. Theorem 1.1 is proven.

CoRollary 1.4. (cf. $[10,1.15])$ Let $P$ be an infinite prime of a field $F$. The following conditions are equivalent:

(1) $P$ is conic and archimedean;

(2) $J_{P}=\{0\}$

(3) There is an isomorphism $\phi$ of $F$ into $\boldsymbol{C}$ such that $\phi^{-1}(\boldsymbol{P})=P$; 
(4) $B_{P}$ is a subfield of $F$.

Proof. (1) implies (2) as $P$ archimedean implies $P^{-}=J_{P}$. implies (3) by Theorem 1.1. (3) implies (4) as $\phi\left(B_{P}\right)=\boldsymbol{R} \cap \phi(F)$. (4) implies $P \subseteq B_{P}$ as each a $\in P$ is equal to $(1-\alpha) \alpha^{-1}$ for $\alpha \in B_{P}$ so $P$ is archimedean; $J_{P}=P^{-}$is an ideal of the field $B_{P}$ so $P^{-}=\{0\}$.

This result yields the remarkable fact first noted by Dubois [10, Theorem IV] that if an infinite prime $P$ of a field $F$ generates a proper subfield of $F$, then $P$ is conic and archimedean, and the (archimedean) order of a maximal orderable subfield of $F$. Such a field must be embeddable in $C$; indeed the subfields of $C$ can be characterized as those fields which contain a conic, archimedean infinite prime [10, Theorem V]. Conic archimedean infinite primes also characterize "number rings":

CoROLlaRY 1.5. The following conditions on a domain $R$ are equivalent:

(1) $R$ is a "number ring", i.e., the quotient field of $R$ is a (possibly infinite) algebraic number field.

(2) $R$ possesses infinite primes, and all its infinite primes are conic.

(3) $R$ possesses infinite primes, and all its infinite primes are archimedean.

Proof. If (1) holds, [10, 4.7] implies all infinite primes of the quotient field $F$ of $R$ are conic, archimedean (as $F$ is algebraic over $\boldsymbol{Q}$, all the primes extend the unique finite prime $\boldsymbol{Q}^{\geq 0}=\boldsymbol{Q} \cap \boldsymbol{P}$ of $\boldsymbol{Q}$ which is conic, archimedean). A fortiori, this holds for all infinite primes of $R$. (2) implies (1) by an example like Example 3 of $\S 4$ : if (1) fails, the non-conic preprime (in fact, infinite prime) $S=\{f(t) \epsilon$ $\boldsymbol{Z}[t] \mid f(0) \geqq 0\}$ of $\boldsymbol{Z}[t]$ ( $t$ transcendental over $\boldsymbol{Z}$ ) extends to a non-conic infinite prime of $R$, contradicting (2). (3) implies (1) by Example 3 of $\S 4$.

We remark that J. E. Schneider in [19] has characterized absolutely integral domains of characteristic zero as domains all of whose finite primes are ideals.

2. Associated primes. To clarify the presentation of this section and the next we introduce the following concept (cf. [18]). By a primed (or localized) field we mean a pair $(F, P)$ where $F$ is a field, $P$ a prime of $F$. We say $(F, P)$ is conic, archimedean, real, etc., if $P$ is conic, archimedean real, etc. A localized place $\phi:(F, P) \rightarrow(K, Q)$ 
is a place $F \rightarrow K$ (in the usual sense) such that $\phi(P) \cong Q$ (where " $\phi(P)$ " means $\phi\left(P \cap D_{\phi}\right), D_{\phi}=$ domain of $\left.\phi\right)$. Theorem 1.1 and [11] imply

Theorem 2.1. (cf. [18]) Each primed field $(F, P)$ determines a localized place $\phi_{P}$ onto the conic archimedean primed field $\left(k_{P}, \boldsymbol{P}_{P}\right)$, the "primed residue field" of $(F, P)$.

Indeed Schneider in [18] shows that the conic archimedean primed fields form a reflective subcategory of the category of primed fields and localized places.

We call two infinite primes $S, P$ of $F$ associated if $J_{P}=J_{S}$ and (in the common residue field) $\boldsymbol{P}_{P}=\boldsymbol{P}_{S}$, or, equivalently, $\operatorname{Arch} P=$ Arch $S$. That is, $P$ and $S$ are associated if they determine the same primed residue field and localized place. Since by Theorem 1.1, Arch $P$ is associated with $P$, each set of associated infinite primes contains a unique archimedean infinite prime; we see below that conversely the set can be recovered from certain groups determined by that archimedean prime.

Let $S$ be a preprime of a commutative ring $R$, let $a \in R$. Define $S: a=\{c \in R \mid c a \in S\}$, write $a={ }_{S} b$ if $S: a=S: b$. Let $\Gamma^{\sharp}(S)$ denote the equivalence classes of $R$ for $=_{s}$, let $\psi_{S}: R \rightarrow \Gamma^{\sharp}(S)$ denote the natural map, and $\Gamma(S)=\Gamma^{\sharp}(S) \backslash \psi_{S}(0)$. By [11, Prop. [2.2] and [15, Prop. 1.1], $\Gamma(S)$ is a group (operation induced by multiplication in $R$ ) if $S$ is a prime or if $\left(A_{S}, S\right)$ is a valuation pair; in the latter case $\Gamma(S)$ is linearly ordered and $\psi_{S}$ "is" the valuation $v_{S}$ associated with the pair (see [15]).

Now let $T$ be the unique archimedean prime in a set of associated infinite primes, let $\phi_{T}:(F, T) \rightarrow\left(k_{T}, T\right)$ be the localized place onto the primed residue field of $T$ (i.e., $\boldsymbol{T}=\boldsymbol{P}_{T}$ ). $T$ determines the following short exact sequence of groups

$$
1 \longrightarrow \Gamma(T) \stackrel{\theta}{\longrightarrow} \Gamma(T) \stackrel{\eta}{\longrightarrow} \Gamma\left(J_{T}\right) \rightarrow 0 .
$$

Here we write $\Gamma\left(J_{T}\right)$ (the value group of the $J_{T}$-valuation) additively; the maps are natural, i.e., $\theta: \psi_{T}\left(a+J_{T}\right) \rightarrow \psi_{T}(a), \eta: \psi_{T}(a) \rightarrow v_{T}(a)$ (where $v_{T}=$ valuation associated with $\left.J_{T}\right)$. Note also that $\eta_{\circ} \psi_{T}=\psi_{J_{T}}=v_{T}$. One can check that $\Gamma(\boldsymbol{T})$ is naturally isomorphic to the group of arguments of the elements of the field $k_{T} \subseteq C$, while $\Gamma(T) \cong F^{\times} / T^{+}$. [One can show that in fact the subgroup $U=T^{+}$of $F^{\times}$determines $T$, and hence (by 2.2 below) the whole set of associated primes; for, $J_{U}=J_{T}$ (recall the definition of $J$ in $\S 0$ ), so $U$ determines $\Gamma(\boldsymbol{T}) \cong$ $\left(A(T) \backslash J_{T}\right) / U, \Gamma(T)$, and $\Gamma\left(J_{T}\right) \cong F^{\times} /\left(A(T) \backslash J_{T}\right)$.]

Below we write $I(\Lambda)$ for the isolated (convex) subgroup of $\Gamma\left(J_{T}\right)$ 
generated by a subgroup $\Lambda$, and let $M_{I(1)}=\left\{a \in F \mid v_{T}(a)>I(\Lambda)\right\}$, the maximal ideal of the valuation pair determined by that isolated subgroup.

THEOREM 2.2. Let $T$ be an archimedean infinite prime of the field $F$. Let $G=G_{T}=\left(A(T) \backslash J_{T}\right) / T^{+}$, the natural image of $\Gamma(T)$ in $\Gamma(T) \cong F^{\times} / T^{+}$, so also the kernel of $\eta$. For a subgroup $H$ of $\Gamma(T)$, let $c(H)=\eta^{-1}(I(\eta(H)))$.

There is a one-to-one correspondence between the infinite primes $P$ of $F$ associated with $T$ and the subgroups $H$ of $\Gamma(T)$ with the property that $H$ is maximal as a subgroup of $c(H)$ which intersects $G$ trivially. The correspondence is given by $P \rightarrow H(P)=\psi_{T}\left(P^{+}\right)$and $H \rightarrow P(H)=\psi_{T}^{-1}(H) \cup M_{I(\eta(H))}$.

REMARK. This theorem says that one gets each associated prime exactly once by the following purely multiplicative procedure. Choose an isolated subgroup $I$ of $\Gamma\left(J_{T}\right)$, then choose a subgroup $H$ of $\eta^{-1}(I)$ maximally disjoint from $G$, and then take $\psi_{T}^{-1}(H) \cup M_{I}$ which is a prime.

Proof. If $P$ is associated with $T$ one checks easily that $H=H(P)$ intersects $G$ trivially. $c(H)$ is easily seen to be $\psi_{T}\left(A_{P^{-}} \backslash P^{-}\right)$(observe that $\psi_{T}\left(A_{P^{-}} \backslash P^{-}\right)$is torsion over $\left.H \cdot G\right)$ and the maximality condition on $H$ now follows from condition (4) (b) of Technical Lemma 1.2 (applied to $S=Q$ ). Conversely if $H \subseteq \Gamma(T)$ is of the type described, $P(H)$ is a prime of $F$ associated with $T$ by (4) of the Technical Lemma (let $P$ replace $S, M_{I(\eta(H))}$ replace $M, T^{+}$replace $G$ ). It is straightforward to check that the correspondences given are inverses of one another.

This theorem translates the study of the infinite primes of $F$ to a study of the complex (and real) places of $F$, their value groups, and the groups $\Gamma(\boldsymbol{T})$ of the conic archimedean infinite primes $\boldsymbol{T}$ on their residue fields. These latter "argument groups" are either the two element group (when the place is real) or are dense in the circle group. The groups $H(P)$ corresponding to infinite primes $P$ associated with a fixed archimedean prime $T$ are natural generalizations of the classical "archimedean equivalence class group" of Baer [1], defined for an order of a field (for $a, b \in P^{+}$define $a$ to be archimedean equivalent to $b$ if there exist $n, m \in N$ with na $-b \in P, m b-a \in P$ ). Indeed, $H(P) \cong v_{P}\left(P^{+}\right)\left(v_{P}=J_{P}\right.$-valuation) and the value group $\Gamma\left(J_{T}\right)=$ $\Gamma\left(J_{P}\right)$ may be thought of as an extension of the archimedean equivalence group to all of $F^{\times}$.

Corollary 2.3. Let $P$ be an infinite prime of the field $F$. (A) The following are equivalent: 
(a) $P$ is an order of $F$,

(b) $P$ is real and the archimedean equivalence class group of $P$ equals $\Gamma\left(J_{P}\right)$,

(c) $P$ is real and, for $T=$ Arch $P, \Gamma(T)=\psi_{T}(P) \cdot G_{T}$;

(B) In general, $P$ real implies $I\left(v_{P}\left(P^{+}\right)\right) / v_{P}\left(P^{+}\right)$is 2-primary torsion;

(C) (cf. [10, Theorem II]) Every real place $\phi$ of $F$ is determined by an order of $F$.

Proof. (A) and (B) are straightforward to check. For (C), let $H$ be a subgroup of $\Gamma(T)$ containing $\Gamma(T)^{2}$ and maximal among subgroups intersecting $G_{T}$ trivially, where $T=\phi^{-1}(P)$, a real archimedean infinite prime of $F$. Then one checks $P(H)$ is the required order, using (A). See $\S 4$ for examples of real infinite primes for which the group in (B) above is nontrivial (so the primes are neither orders nor modes).

We now consider associated primes in field extensions and apply this to prove an existence theorem needed in $\S 3$. Recall that an infinite prime of a ring can always be extended to an overring (cf. [11]) and that by Dubois' result (Prop. 1.3) a conic infinite prime of a field can be extended to a conic infinite prime of an overfield.

Let $F \cong K$ be fields, $P, S$ primes of $F, K$ respectively. If $S$ extends $P$ (i.e., $S \supseteqq P$ ) then $S^{+}, S^{-}, J_{S}$, Arch $S$, etc., intersect $F$ in $P^{+}, P^{-}, J_{P}$, Arch $P$, etc., respectively, and all the groups (cf. 2.2) determined by $P$ are naturally imbedded in those determined by $S$.

Proposition 2.4. Let $F \subseteq K$ be fields, $P, S$ infinite primes of $F, K$ respectively. Then $S \supseteqq P$ if and only if $J_{S} \cap F=J_{P}, \boldsymbol{P}_{S} \supseteqq \boldsymbol{P}_{P}$, $H(S) \supseteqq H(P)($ in $\Gamma($ Arch $S))$ and $I(\eta(H(S))) \cap \Gamma\left(J_{P}\right)=I(\eta(H(P)))$. When $K$ is algebraic over $F$, the last condition may be replaced by " $I(\eta(H(S)))$ is the torsion closure of $I(\eta(H(P)))$ in $\Gamma\left(J_{S}\right)$ ".

Proof. The "only if" part is straightforward. For the "if" part, $S^{-} \supseteqq P^{-}$follows from $J_{S} \cap F=J_{P}$ and the condition on isolated subgroups, while $S^{+} \supseteqq P^{+}$follows from $\boldsymbol{P}_{S} \supseteqq \boldsymbol{P}_{P}$ and $H(S) \supseteqq H(P)$. The last assertion holds as $\Gamma\left(J_{S}\right)$ is then torsion over $\Gamma\left(J_{P}\right)$.

Proposition 2.5. Let $P$ be an infinite prime of the field $F$. Let $K \supseteqq F$ be a field, $w$ a valuation on $K$ extending $v_{P}$ on $F, T$ a conic archimedean infinite prime of $k_{w}$. If $\boldsymbol{T} \supseteqq \boldsymbol{P}_{P}$ then there exists an infinite prime $S$ of $K$ with $S \supseteq P$ such that $w=v_{S}$ (i.e. $J_{S}$ determines w) and $\boldsymbol{P}_{S}=\boldsymbol{T}$.

Moreover, if $\boldsymbol{T}$ is real and $\Gamma_{w}=\Gamma_{v_{P}}\left(=\Gamma\left(J_{P}\right)\right)$, then $S$ is unique. 
Proof. Let $T=\phi_{w}^{-1}(T)$, an archimedean infinite prime of $K$. The hypotheses imply $T \cap F=\operatorname{Arch} P$ and that $H(P)$ is a subgroup of $\Gamma(T)$ disjoint from $G_{T}$. Let $H \supseteqq H(P)$ be maximal among subgroups of $\eta_{T}^{-1}\left(I\left(v_{T}\left(A_{P^{-}} \backslash P^{-}\right)\right)\right)$intersecting $G$ trivially, then $S=P(H)$ is as required. The uniqueness assertion follows from the fact that in that case, $\Gamma(T)=\Gamma(\operatorname{Arch} P)$ in the embedding, so $H(P)$ itself is already maximal.

3. Ultracompletions. Let $(F, P)$ be a primed field. We call a primed field $(K, S)$ an extension of $(F, P)$ if $K \supseteqq F$ and $S \supseteqq P$. For a primed field $(F, P)$ we shall define an extension $(F, P)$ (in general non-unique) which we will call an ultracompletion of $(F, P)$; this construction will generalize the completion of an algebraic number field at a prime spot. First, we say that a primed field extension $(K, S)$ of $(F, P)$ is immediate if the following conditions are satisfied: $\Gamma\left(J_{S}\right)=\Gamma\left(J_{P}\right)$, and $k_{S}=k_{P}$ when $P$ is a finite prime, or $k_{S}$ is real if $k_{P}$ is real when $P$ is infinite. (Note $S \supseteqq P$ implies $S$ is a finite prime if and only if $P$ is.) When $P$ is finite above, $K$ with $v_{S}$ is an immediate extension of $F$ with $v_{P}$, in the sense of valuation theory. We say a primed field $(F, P)$ is ultracomplete if it has no proper immediate extensions. Finally, we say $(K, S)$ is an ultracompletion of $(F, P)$, if $(K, S)$ is an ultracomplete, immediate extension of $(F, P)$ or, equivalently, a maximal immediate extension of $(F, P)$.

THEOREM 3.1. Every primed field $(F, P)$ has an ultracompletion $(\hat{F}, \hat{P})$. A primed field $(\hat{F}, \hat{P})$ is ultracomplete if and only if $F$ is linearly compact for the valuation $v_{\hat{P}}$, and, $k_{\hat{P}} \cong C$ if $\hat{P}$ is complex or $\boldsymbol{R}$ if $\hat{P}$ is real. Ultracompletions of finite or complex infinite primes are not necessarily unique up to isomorphism. Ultracompletions of real infinite primes are unique up to isomorphism.

Proof. The first assertion is classical (cf. [13]) for finite primes. If $(F, P)$ is infinite, Krull's embedding lemma of [13] yields a set embedding of $F$ into the well ordered power series field $W\left(\boldsymbol{C}, \Gamma\left(J_{P}\right)\right)$ over the value group $\Gamma\left(J_{P}\right)$ with coefficients in $C$, giving the cardinality restriction permitting Zorn's lemma to apply. For the second sentence, maximality with respect to immediate primed field extensions implies (using Prop. 2.5 in the infinite case) maximality of $\hat{F}$ with respect to the valuation $v_{\hat{P}}$, and hence that $\hat{F}$ is linearly compact at $v_{P}$ (see [2]). $\quad k_{\hat{P}} \cong \boldsymbol{C}$ or $\boldsymbol{R}$ (according as $\hat{\boldsymbol{P}}$ is complex or real) follows from Prop. 2.5 and [17, Lemma 19, p. 218]. Nonuniqueness in the finite case is shown by Example 5; Example 2 yields examples showing nonuniqueness in the complex infinite case. Uniqueness when $\hat{P}$ is real follows from Kaplansky's Theorem ([12, Theorem 7]), the unique ordering in $\boldsymbol{R}$, and the uniqueness in Prop. 2.5 (Note that Kaplansky's 
hypothesis on the existence of arbitrary $n$th roots in the residue field is not needed, since there is no change in the value group in passing to an ultracompletion.)

Because of the nonuniqueness of ultracompletions, it is reasonable to consider the set of all ultracompletions $(\hat{F}, \hat{P})$ of a primed field $(F, P)$ (that a representative set of these can be chosen from the class of all such follows from the Krull embedding noted in the proof of 3.1). We shall examine the set of ultracompletions in relation to squares in the field. Specifically, we shall describe the "squares kernel", i.e., the set of elements of $F$ which become squares in every ultracompletion. We shall use without explanation the techniques of the paper [4], the basic definitions of which we now outline.

Let $v$ be a valuation on a field $F$, with value group $\Gamma_{v}$. Define a linearly ordered set $\Lambda_{v}$ by

$$
\Lambda_{v}=\left\{0^{*}\right\} \cup\left\{\alpha \in \Gamma_{v} \mid \alpha \geqq 0\right\}
$$

where $0^{*}$ is a formal symbol, $\Lambda_{v} \cap \Gamma_{v}$ inherits its order from $\Gamma_{v}$, and $0^{*} \leqq \alpha$ for all $\alpha \in \Lambda_{v}$. The map $v^{\times}: F^{\times} \rightarrow \Lambda_{v} \cup\{\infty\}$ defined by $v^{\times}(a)=0^{*}$ if $v(a) \neq 0, v^{\times}(a)=v(1-a)$ if $v(a)=0$, is a group valuation on $F^{\times}$(i.e. $v(a)=\infty$ if and only if $a=1, v^{\times}(a)=v^{\times}\left(a^{-1}\right)$, and $v^{\times}(a b) \geqq$ $\left.\min \left\{v^{\times}(a), v^{\times}(b)\right\}\right)$. For $\alpha \in \Lambda_{v}$, let $F_{\alpha}^{\times}=\left\{a \in F^{\times} \mid v^{\times}(a) \geqq \alpha\right\}$. Define a $\Lambda_{v}$-graded group $\Delta^{s q}(F)$ (i.e., a family of groups indexed on $\Lambda_{v}$ ) by

$$
\begin{aligned}
\Delta^{s q}(F)_{\alpha} & =\Gamma_{v} / 2 \Gamma_{v} & & \text { if } \alpha=0^{*} \\
& =k_{v}^{\times} / k_{v}^{\times 2} & & \text { if } \alpha=0 \\
& \left.=k_{v}^{+} \text {(additive group of } k_{v}\right) & & \text { if } 0<\alpha<v(4), \alpha \notin 2 \Gamma_{v} \\
& =k_{v}^{+} /\left\{a^{2}-a \mid a \in k_{v}\right\} & & \text { if } \alpha=v(4)>0 \\
& =\text { the trivial group } & & \text { otherwise. }
\end{aligned}
$$

It is shown in [4] that when $F$ is linearly compact at $v, F^{\times} / F^{\times 2}$ is isomorphic to the well ordered product $\boldsymbol{H}_{\alpha \in A_{v}} \Delta^{s q}(F)_{\alpha}$ (i.e., the subgroup of $\Pi \Delta^{s q}(F)_{\alpha}$ of elements with support a well ordered subset of $\Lambda_{v}$ ). This theorem yields a calculation of the "squares kernel". Define:

$$
\begin{aligned}
K^{s q}(F, P) & =(\operatorname{Arch} P)^{+} \cdot F^{\times 2} \text { if } P \text { is real infinite } \\
& =F_{0}^{\times} \cdot F^{\times 2} \quad \text { if } P \text { is complex infinite } \\
& =\cup\left\{F_{\alpha}^{\times} \cdot F^{\times 2} \mid \alpha \in \Lambda_{v}, \beta \geqq \alpha \text { implies } \Delta^{s q}(F)_{\alpha} \text { trivial }\right\} \\
& \text { if } P \text { is finite. }
\end{aligned}
$$

(Note: $K^{s q}(F, P)=1+4 \cdot J_{P}$ when $P$ is finite, and $k_{P}$ has a quadratic extension.) 


\section{$\bigcap\left\{\hat{F}^{\times 2} \cap F^{\times} \mid(\hat{F}, \hat{P})\right.$ an ultracompletion of $\left.(F, P)\right\}=K^{s q}(F, P)$}

Proof. Recalling that $k_{\hat{P}} \cong \boldsymbol{R}$ (resp. $\boldsymbol{C}$ ) when $P$ is real (resp. complex) infinite, one can check easily that Theorem 1.1 of [4] implies $K^{s q}(F, P)$ is contained in $\hat{F}^{\times 2}$ for any ultracompletion $(\hat{F}, \hat{P})$, in all cases. Conversely, suppose $a \notin K^{s q}(F, P)$. If $P$ is complex infinite this means $v(a) \notin 2 \Gamma\left(J_{P}\right)$ and $a \notin \hat{F}^{\times 2}$ for any ultracompletion. If $P$ is real infinite this means either $v(a) \notin 2 \Gamma\left(J_{P}\right)$ or for some $c \in F^{\times}, \phi_{P}\left(a c^{2}\right)<0$ in $k_{P}$, hence in any $k_{\hat{P}}$, so $a \notin$ any $\hat{F}^{\times 2}$. If $P$ is finite there must exist $\beta \in \Lambda_{v}$ with $\beta>\left\{v^{\times}\left(a c^{2}\right) \mid c \in F^{\times}\right\}$, and with $\Delta^{s q}(F)_{\beta} \neq 1$; let $v^{\times}(b)=v^{s q}\left(b F^{\times 2}\right)=\beta$. Then $\left\{v^{\times}\left(a b \cdot c^{2}\right) \mid c \in F^{\times}\right\}$has no maximum, but is bounded above by $\beta$, which must be $\leqq v(4)$. Hence by [4, Remark 5.2], the extension $F^{\prime}=F \sqrt{a b}$ over $F$ has $e=f=$ $g=1$. Let $P^{\prime}$ be the unique prime on $F^{\prime}$ extending $P$, then $\left(F^{\prime}, P^{\prime}\right)$ is an immediate extension of $(F, P)$, and, in $F^{\prime \prime}, v^{s q}\left(a F^{\prime \times 2}\right)=\beta$, (as $\left.a \cdot(b / \sqrt{a b})^{2}=b\right)$ so that $a$ is not a square in any ultracompletion $\left(\hat{F}^{\prime}\right.$, $\left.\hat{P}^{\prime}\right)$ of $\left(F^{\prime \prime}, P^{\prime}\right)$. However, any such $\left(\hat{F}^{\prime}, \hat{P}^{\prime}\right)$ is also an ultracompletion of $(F, P)$, and thus $a \notin \hat{F}^{\times 2}$ for some ultracompletion of $(F, P)$.

We end this section by indicating an aspect of the ultracompletions $(\hat{F}, \hat{P})$ of a primed field $(F, P)$ which is unique (except when $P$ is complex infinite with $\left[k_{P}: \boldsymbol{P}_{P}-\boldsymbol{P}_{P}\right]>2$ as in Examples 1 and 2). Each $F$ with $v_{P}$ is a valued field, so the field $\Delta F$ of [6] is defined (and is the field of real or complex Laurent series over $\Gamma\left(J_{P}\right)$ if $P$ is real or complex). In the notation of [6] let $\Delta P$ be all $b \in \Delta F$ such that $b$ has a representation $\sum b_{i} t^{i}+\mathfrak{b}_{F}$ with $b_{j} \in P$ where $j=\Delta\left(v_{P}\right)(b)$. One can show $\Delta P$ is a prime of $\Delta F . \quad F$ and $\Delta F$ have very similar properties (see [6]). $(\Delta F, \Delta P)$ can be constructed from $(F, P)$ alone (except in the case noted above) and so is unique.

4. Examples. This section contains examples illustrating some pathology of complex conic archimedean primes, of ultracompletions, and of the nonfield case, and an application of Theorem 2.2 to contruction of infinite primes in $\boldsymbol{Q}(x)$.

EXAMPLE 1. Let $P$ be the nonnegative rationals, the unique (conic, archimedean) infinite prime of $\boldsymbol{Q}$. Let $f_{p}(x)$ be a polynomial of degree a prime number $p \geqq 3$, irreducible over $\boldsymbol{Q}$, and possessing exactly two nonreal roots (see [3, p. 130-131] for a construction of such polynomials). Let $K$ be a splitting field of $f_{p}(x)$ over $\boldsymbol{Q}$, let $G=\operatorname{Gal}(K / \boldsymbol{Q})$. $G$, as a permutation group on the roots of $f_{p}$, contains a $p$-cycle $\sigma$ (as $p \mid[K: \boldsymbol{Q}]=(G: 1)$ ) and a transposition, namely complex conjugation $\omega$. Hence $G \cong \sum_{p}$, the symmetric group on $p$ letters. Let $F=K^{\langle\sigma\rangle}$, the fixed field of the cyclic subgroup $\langle\sigma\rangle$ of order $p$ generated by $\sigma$. Then $[F: P-P]=[F: Q]=(p-1) !$, and moreover $P$ is an infinite 
prime of the larger field $F$. [Suppose on the contrary that $T \supsetneqq P$ is an infinite prime of $F ; T$ is conic and archimedean as $F$ is algebraic over $\boldsymbol{Q}$ (cf. [10,4.7]), so $T-T$ is isomorphic to a subfield of $\boldsymbol{R}$, say by $\tau \in G$. Hence $\omega$ leaves $\tau(T-T)$ elementwise fixed, and therefore $T-T \subseteq F=K^{\sigma}$ is fixed (elementwise) by both the $p$-cycle $\sigma$ and the transposition $\tau^{-1} \omega \tau$. Hence the subgroup of $G$ fixing $T-T$ is all of $G, T-T=Q$, and $T=P$, a contradiction.]

REMARK. This example and the following one show that for a conic archimedean infinite prime $P$ of a field $F,[F: P-P]=\left[F: B_{P}\right]$ may be quite large, even infinite. Whenever $[F: P-P]=2 m>2$, the $m$ conjugate pairs of embeddings of $(F, P)$ into $(\boldsymbol{C}, \boldsymbol{P})$ yield $m$ nonisomorphic ultracompletions of $(F, P)$.

ExAMPLE 2. Let $P$ be an archimedean order of a field $F$, and suppose that $P \nsubseteq F^{\times 2}$. Let $a \in P \backslash F^{\times 2}$, let $\alpha_{0}=-a$, in general for $n \geqq 1$ let $\alpha_{n}^{2}=\alpha_{n-1}$ (in some algebraic closure of $F$ ). Let $F_{n}=F\left(\alpha_{n}\right)$. Then $\left[F_{n}: F\right]=2^{n}$ for all $n$, and $P$ is an infinite prime of $F_{\infty}=\bigcup_{n=1}^{\infty} F_{n}$ (so a fortiori of every $F_{n}$ ). [observe $\pm \alpha_{n} \notin F_{n}^{2}$ for $n \geqq 1, i \notin F_{n}$ for any $n\left(i^{2}=-1\right)$, and then that the $F_{n}$ are the only intermediate fields between $F_{\infty}$ and $\left.F.\right]$

ExAmple 3. This shows Corollary 1.4 false for $F$ not a field and completes proving Corollary 1.5. Let $R=Z[x], x$ an indeterminate. Order $R$ by: $f(x)>0$ if and only if the leading coefficient of $f$ is positive. Let $P$ be the set of nonnegative elements of $R$ under this ordering. $P$ is a prime of $R$, for $P \supseteqq Z^{+}=$nonnegative integers, a prime of $\boldsymbol{Z}$, so $P \cap \boldsymbol{Z}=Z^{+}$, and if $f \in R \backslash P, f \notin Z$, then $-f-1 \in P$, so $-1=f+(-f-1)$, and $f$ is in no preprime containing $P$. $P$ is non archimedean as $n-x \notin P$ for any $n \in N$, but $J_{P}=\{0\}$.

ExAmple 4. We indicate how Theorem 2.2 can be used to consruct primes by constructing some infinite primes of $\boldsymbol{Q}(x)$; for more examples, see [20]. Let $f(x)$ be an irreducible polynomial over $\boldsymbol{Q}$, let $P_{0}$ be an infinite prime of $\boldsymbol{Q}[x] /(f(x)) \cong \boldsymbol{Q}(\alpha), \alpha$ a root of $f$. Let $U_{f}=\left\{g(x) / h(x) \mid g(\alpha) / h(\alpha) \in P_{0}\right\}$ (where " $\varepsilon P_{0}$ " means "defined and in $P_{0}$ "). Let $J=\{g(x) / h(x) \mid g(\alpha) / h(\alpha)$ is defined and $=0\}$, the maximal ideal of the $f$-adic valuation. If there exist $n \in N$ and $c(x) \in A_{J} / J$ such that $c(x) \cdot U_{f} \notin \bigcup\left\{\Gamma\left(P_{0}\right)^{p} \mid p\right.$ is a prime number, $\left.p \mid n\right\}\left(\right.$ recall $\left.\Gamma\left(P_{0}\right) \cong\left(A_{J} \mid J\right) / U_{f}\right)$ then the set

$$
\{0\} \cup\left\{U_{f} \cdot\left(c(x) \cdot f^{n}\right)^{k} \mid k \in Z\right\}
$$

is an infinite prime $P=P(f, c)$ of $\boldsymbol{Q}(x)$, with $J_{P}=J, k_{P} \cong \boldsymbol{Q}(\alpha), \boldsymbol{P}_{P}=$ 
$P_{0}$. In fact, when $\operatorname{deg} f>1$, every non-archimedean prime containing the "generating cone" $U_{f}$ has the above form. In the special case that $P_{0}$ is real, i.e., an order of $\boldsymbol{Q}(\alpha)$, taking $n=2^{m}$ for $m \geqq 1$ and $c(x)=-$ 1 yields a real conic non-archimedean prime which is not an order, hence not a mode; thus Theorem 1.1 extends Dubois' results even for real infinite primes.

REMARK. The above construction, plus analogous ones for $\operatorname{deg} f=$ 1 and for $f=1 / x$ yields all the conic infinite primes of $\boldsymbol{Q}(x)$ which generate $\boldsymbol{Q}(x)$ as a field; the non-conic infinite primes are the Arch $P$ 's for the the above $P$ 's. The other infinite primes are characterized by [10, Thm. IV]: conic, archimedean primes generating proper subfields. The method of Example 2 yields examples of these, e.g. if $P$ is an archimedean order of $\boldsymbol{Q}\left(x^{2^{n}}\right)$ such that $x^{2^{n}} \notin P$, then $P$ is an infinite prime of $\boldsymbol{Q}(x)$. Ron Brown in [5] has described all finite primes of $\boldsymbol{Q}(x)$.

EXAMPLE 5. We construct nonisomorphic ultracompletions of a primed field $(F, P)$ : Let $F_{n}=\boldsymbol{Q}\left(\alpha_{n}\right)$ where $\alpha_{0}=2, \alpha_{n}^{2}=\alpha_{n-1}$ for $n \geqq 1$, let $F=\bigcup_{n=1}^{\infty} F_{n}$. Then there is a unique prime $P$ of $F$ extending the prime $P(2)$ of $\boldsymbol{Q}(P(2)$ is the maximal ideal of the 2-adic valuation ring of $\boldsymbol{Q}$, see [11]). Let $v=v_{P(2)}$. Then (see [4] for notation) the set $\left\{v^{\times}\left(-1 \cdot c^{2}\right) \mid c \in F^{\times}\right\}$has no maximum but is bounded above by $v(4)=v^{\times}(5)=\max \left\{v^{\times}\left(5 \cdot c^{2}\right) \mid c \in F^{\times}\right\}$, so also the set $\left\{v^{\times}\left(-5 \cdot c^{2}\right) \mid c \in F^{\times}\right\}$ has no maximum but is bounded above by $v(4)$. Hence $F \sqrt{-1}$ and $F \sqrt{-5}$ are immediate extensions of $F$ (with the unique prime extending $P$ ), but -1 is not a square in any ultracompletion of $F \sqrt{-5}$ (as $\left.-1 \cdot(\sqrt{-5 /}(-1))^{2}=5\right)$ while -1 is a square in $F \sqrt{-1}$, so in any ultracompletion of $F \sqrt{-1}$. Since any ultracompletion of $F \sqrt{-1}$ or $F \sqrt{-5}$ is one of $F$, we have our example (in proving this, show $\max v_{n}^{\times}\left(-1 \cdot F_{n}^{\times}\right)=\left(1+\sum_{k=1}^{n} 2^{-k}\right) \cdot v_{n}(2)$ for all $n$ and apply [4, Remark $5.2])$.

REMARK. This example can be extended to show the existence of ultracompletions with nonisomorphic Witt-Grothendieck rings of equivalence classes of quadratic forms.

Note. MacLane's construction in [14, Example II, p. 381] gives an example of a primed field $(F, P)$ with nonisomorphic ultracompletions, in which the prime $P$ is finite, $k_{P}=Z_{p}$, and the value group $\Gamma\left(J_{P}\right)$ is discrete rank two. 


\section{APPENDIX}

The lemma below contains the crucial trick inspired by Dubois' examples in [10, §2]. Throughout this appendix we write a.c. for additively closed, m.c. for multiplicatively closed.

LeMma A. Let $(A, M)$ be a valuation pair of $F$.

(1) If $G$ is an a.c. subgroup of $A \backslash M$, then $G+M=G(1+M)$ is also an a.c. subgroup.

(2) If $H$ is a subgroup of $F^{\times}$and $H \supseteqq 1+M$ then $H$ is a.c. if and only if $H \cap(A \backslash M)$ is a.c.

Proof. (1): Elementary valuation ring theory. (2): If $H$ is a.c., suppose $h_{1}, h_{2} \in H \cap(A \backslash M)$ but $h_{1}+h_{2}$ is not, then $h_{1}+h_{2}=\alpha \in M$ and $0=h_{1}+\left(-h_{1}\right)=h_{1}+h_{2}\left(1-h_{2}^{-1} \alpha\right) \in H+H(1+(A \backslash M) M) \leqq H, a$ contradiction. Conversely, let $H \cap(A \backslash M)$ be a.c. and $h_{1}, h_{2} \in H$. Supposing $h_{1}^{-1} h_{2} \in A$ without loss, we have $h_{1}^{-1} h_{2}$ in $H \cap(A \backslash M)$ or in $M$, so $1+h_{1}^{-1} h_{2} \in H \cap(A \backslash M)$ and $h_{1}+h_{2}=h_{1}\left(1+h_{1}^{-1} h_{2}\right) \in H$.

Proof of Technical Lemma 1.2. (1) implies (3): $\left(A_{S^{-}}, S^{-}\right)$is a valuation pair and $S^{+}$is an a.c. subgroup of $F^{\times}$by [11, Props. 2.5, 1.6]. Let $(A, M)$ be a valuation pair with $M \supseteqq S^{-} \cdot M \cong J_{S}$ is equivalent to $1+M \subseteq S^{+}$by definition of $J_{S}^{2}$ which implies $S^{+} \cap(A \backslash M)$ is a.c. by $A(2)$, so (a) implies (b). If (b) holds and $H$ is a maximal a.c. subgroup of $A \backslash M$ with $H \supseteqq S^{+} \cap(A \backslash M)$, then $H \supseteqq 1+M$ by $A(1)$ and maximality. Hence $H: S=H \cdot S^{+} \cup S^{-}$is a preprime $\supseteqq S$, so $=S$, and $H=S^{+} \cap$ $(A \backslash M)$, proving (c). $\quad\left(H \cdot S\right.$ is a preprime since $H \subseteq A \subseteq A_{S^{-}}, H \cdot S^{+}$ and $S^{-}$are both a.c. and m.c., while $H \cdot S^{+} \cdot S^{-} \subseteq S^{-}, H \cdot S^{+}+S^{-}=$ $H \cdot S^{+}\left(1+S^{-}\right) \subseteq H S^{+}$, and $-1 \in H \cdot S$ implies $-1 \in H \cdot S \cap(A \backslash M)=H$, a contradiction). (c) implies (a) as $A(1)$ and maximality imply $1+M \subseteq$ $S^{+} \subseteq S$.

(3) implies (4): Let $(A, M)=\left(A_{S^{-}}, S^{-}\right)$

(4) implies (2): With $(A, M)$ the given valuation pair, let $H \supseteqq$ $S^{+}$be a maximal a.c. subgroup of $A_{S^{-}} \backslash S^{-} . \quad G=S^{+} \cap(A \backslash M) \supseteqq 1+M$ (as before) so $H \supseteqq 1+M$ and $H \cap(A \backslash M)=G$ (as $H \cap(A \backslash M)$ is a.c. by $A(2), G$ is maximal), hence $H=S^{+}$by (b) of (4), proving (2). implies (1): $S$ is a.c. and m.c. since both $S^{+}$and $S^{-}$are, and $S^{+} \cdot S^{-}$, $\subseteq S^{-}, S^{+}+S^{-}=S^{+}\left(1+S^{-}\right) \subseteq S^{+}$(as $1+S^{-} \subseteq S^{+}$by maximality), and $-1 \epsilon$ $S$ would imply $0=-1+1 \in S^{+}+S^{+} \subseteq S^{+}$, a contradiction, so $S$ is a preprime. Let $T \supseteqq S$ be a preprime and suppose $a \in T \backslash S$. If $a \notin$ $A_{S^{-}},-1=a \cdot(-a)^{-1} \in a \cdot S^{-} \subseteq T$, a contradiction. If $a \in A_{S^{-}}$, so in $A_{S^{-}} \mid S^{-}$, let $H=\left\{\sum_{i=0}^{n} s_{i} a^{i} \mid s_{i} \in S^{+} \cup\{0\}\right.$, some $\left.s_{i} \neq 0, n \geqq 0\right\} ; H$ is a.c. and m.c. If $H \cap S^{-}=\varnothing$ then $\left\{h_{1} h_{2}^{-1} \mid h_{i} \in H\right\}$ would be an a.c. subgroup of $A_{S^{-}} / S^{-}$ properly containing $S^{+}$, a contradiction. So, $\sum_{i=0}^{n} s_{i} a^{i}=\alpha \in S^{-}$and we

$21+M \subseteq S+$ as $1+m \in S-\Rightarrow-1=(1+2 m)+2(-1-m) \in S+S \subseteq S$, contra. 
may assume $n$ least so $s_{0} \neq 0, n \geqq 1$. But then $-1=\sum_{i=1}^{n}\left(s_{0}-\right.$ $\alpha)^{-1} s_{i} \alpha^{i} \in T$ (as $s_{0}-\alpha \in S^{+}$), a contradiction. Thus $S$ is a prime, (1) holds.

The last assertion of the lemma follows as (1) implies (2) and (3) hold.

\section{REFERENCES}

1. R. Baer, Uber nichtarchimedisch geordnete korper, S-B Heidelberger Akad. Wiss. Math. Natur. Kl., 8 (1927), 3-13.

2. N. Bourbaki, Algebre Commutative, Chap. 6 Fasc. XXX, Act. Sci et Indust. \#1308, Hermann, Paris, 1964.

3. R. Brauer, Notes on Field Theory, Harvard University, 1958.

4. Ron Brown and H. D. Warner, Quadratic extensions of linearly compact fields, Trans. Amer. Math. Soc., 162 (1971), 326-373.

5. Ron Brown, Thesis, University of Oregon, 1968.

6. Ron Brown and D. K. Harrison, Tamely ramified extensions of linearly compact fields, J. Algebra, 15 (1970), 371-375.

7. D. W. Dubois, On partly ordered fields, Proc. Amer. Math. Soc., 7 (1956), 918-930. 8. —_, A note on David Harrison's theory of preprimes, Pacific J. Math., 21 (1967), 15-19.

9. - Second note on David Harrison's theory of preprimes, Pacific J. Math., 24 (1968), 57-68.

10. - Infinite prime and ordered fields, Dissertationes Math. (Rozprawy Math.), 69 (1971).

11. D. K. Harrison, Finite and infinite primes for rings and fields, Memoir $\$ 68$ of Amer. Math. Soc., Providence, R. I., 1966.

12. I. Kaplansky, Maximal fields with valuations, Duke J. Math., 9 (1942), 303-321.

13. W. Krull, Allgemeine Bewertungstheorie, J. fur Reine und. Angew. Math., 167 (1932), 160-196.

14. Saunders MacLane, The uniqueness of the power series representation of fields with valuations, Annals of Math. 2nd series, 39 (1938), 370-382.

15. M. E. Manis, Valuations on a commutative ring, Proc. Amer. Math. Soc., 20 (1969), 193-198.

16. H. G. Rutherford, Characterizing primes in some noncommutative rings, Pacific J. Math., 27 (1968), 387-392.

17. O. F. G. Schilling, Theory of valuations, Math. Surveys No. 4, Amer. Math. Soc., New York, 1950.

18. J. E. Schneider, Thesis, University of Oregon, 1968.

19. - A note on the theory of primes, Pacific J. Math., 30 (1969), 805-810,

20. H. D. Warner, Thesis, University of Oregon, 1968.

21. —_, Finite primes in simple algebras, Pacific J. Math., 36 (1971), 245-265.

Received October 27, 1971. The first author was supported by NSF grants $\#$ GP-5340 and $\#$ GP-32842. The second author was supported by NSF grant $\# G P-5340$, and an NSF terminal graduate fellowship.

The main part of this paper appeared in the thesis of the second author, written at the University of Oregon under the supervision of the first author.

UNIVERSITY OF OREGON

AND

VANDERBILT UNIVERSITY 


\section{PACIFIC JOURNAL OF MATHEMATICS}

EDITORS

\author{
H. SAMELSON \\ Stanford University \\ Stanford, California 94305 \\ C. R. HOBBY \\ University of Washington \\ Seattle, Washington 98105
}

\author{
J. DUGUNDJI \\ Department of Mathematics \\ University of Southern California \\ Los Angeles, California 90007 \\ RICHARD ARENS \\ University of California \\ Los Angeles, California 90024
}

\section{ASSOCIATE EDITORS}

E. F. BECKENBACH

B. H. NeUManN

F. WOLF

K. YosHIDA

\section{SUPPORTING INSTITUTIONS}

UNIVERSITY OF BRITISH COLUMBIA

CALIFORNIA INSTITUTE OF TECHNOLOGY

UNIVERSITY OF CALIFORNIA

MONTANA STATE UNIVERSITY

UNIVERSITY OF NEVADA

NEW MEXICO STATE UNIVERSITY

OREGON STATE UNIVERSITY

UNIVERSITY OF OREGON

OSAKA UNIVERSITY
UNIVERSITY OF SOUTHERN CALIFORNIA

STANFORD UNIVERSITY

UNIVERSITY OF TOKYO

UNIVERSITY OF UTAH

WASHINGTON STATE UNIVERSITY UNIVERSITY OF WASHINGTON

AMERICAN MATHEMATICAL SOCIETY NAVAL WEAPONS CENTER 


\section{Pacific Journal of Mathematics}

\section{Vol. 45, No. $1 \quad$ September, 1973}

William George Bade, Complementation problems for the Baire classes .......... 1

Ian Douglas Brown, Representation of finitely generated nilpotent groups ........ 13

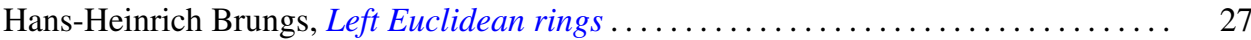

Victor P. Camillo and John Cozzens, A theorem on Noetherian hereditary rings ..... 35

James Cecil Cantrell, Codimension one embeddings of manifolds with locally flat

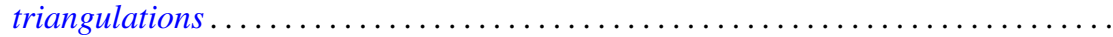

L. Carlitz, Enumeration of up-down permutations by number of rises . . . . . . . . . .

Thomas Ashland Chapman, Surgery and handle straightening in Hilbert cube

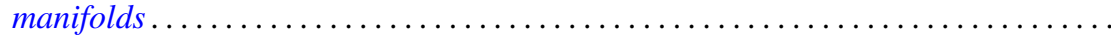

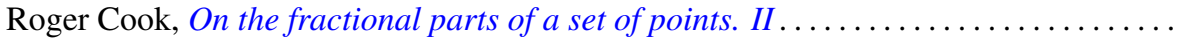

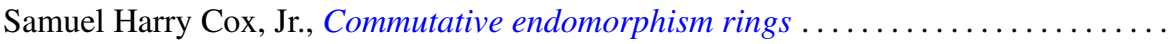

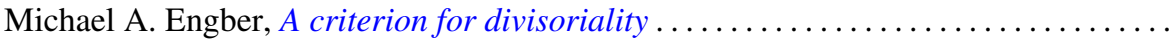

Carl Clifton Faith, When are proper cyclics injective . . . . . . . . . . . . . . 97

David Finkel, Local control and factorization of the focal subgroup . . . . . . . . . 113

Theodore William Gamelin and John Brady Garnett, Bounded approximation by

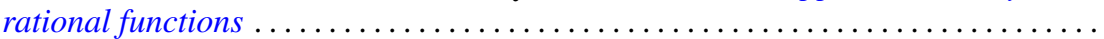

Kazimierz Goebel, On the minimal displacement of points under Lipschitzian

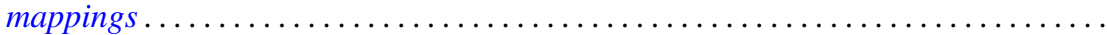

Frederick Paul Greenleaf and Martin Allen Moskowitz, Cyclic vectors for representations associated with positive definite measures: nonseparable

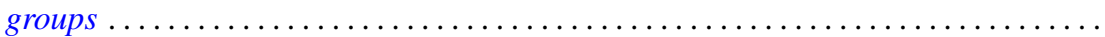

Thomas Guy Hallam and Nelson Onuchic, Asymptotic relations between perturbed linear systems of ordinary differential equations .

David Kent Harrison and Hoyt D. Warner, Infinite primes of fields and completions. .

James Michael Hornell, Divisorial complete intersections . ......

Jan W. Jaworowski, Equivariant extensions of maps ..............

John Jobe, Dendrites, dimension, and the inverse arc function .. .

Gerald William Johnson and David Lee Skoug, Feynman integrals of non-factorable

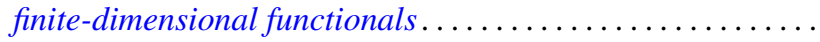

Dong S. Kim, A boundary for the algebras of bounded holomorphic functions ...... 269

Abel Klein, Renormalized products of the generalized free field and its derivatives ... 275

Joseph Michael Lambert, Simultaneous approximation and interpolation in $L_{1}$ and

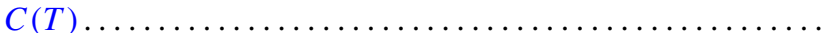

Kelly Denis McKennon, Multipliers of type $(p, p)$ and multipliers of the group

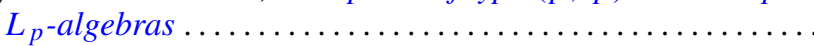

William Charles Nemitz and Thomas Paul Whaley, Varieties of implicative

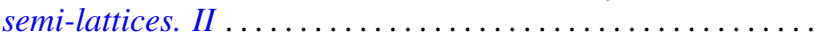

Donald Steven Passman, Some isolated subsets of infinite solvable

Norma Mary Piacun and Li Pi Su, Wallman compactifications on E-completely

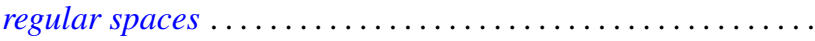

Jack Ray Porter and Charles I. Votaw, $S(\alpha)$ spaces and regular Hausdorff extensions....

Gary Sampson, Two-sided $L_{p}$ estimates of convolution transforms .

Ralph Edwin Showalter, Equations with operators forming a rig
Raymond Earl Smithson, Fixed points in partially ordered sets .

Victor Snaith and John James Ucci, Three remarks on symmetric products and

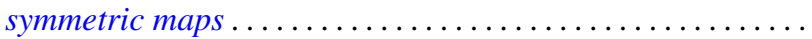

